\title{
Functional Architecture and Encoding of Tactile Sensorimotor Behavior in Rat Posterior Parietal Cortex
}

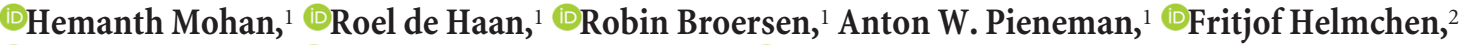 \\ 이ochen F. Staiger, ${ }^{3}{ }^{\oplus}$ Huibert D. Mansvelder, ${ }^{1}$ and ${ }^{\circledR}$ Christiaan P.J. de Kock $^{1}$ \\ ${ }^{1}$ Department of Integrative Neurophysiology, Center for Neurogenomics and Cognitive Research, Neuroscience Campus Amsterdam, VU University \\ Amsterdam 1081 HV, The Netherlands, ${ }^{2}$ Brain Research Institute, University of Zurich, 8057 Zurich, Switzerland, and ${ }^{3}$ Institute for Neuroanatomy, \\ University Medical Center Göttingen, Georg-August-University, D-37075 Göttingen, Germany
}

The posterior parietal cortex (PPC) in rodents is reciprocally connected to primary somatosensory and vibrissal motor cortices. The PPC neuronal circuitry could thus encode and potentially integrate incoming somatosensory information and whisker motor output. However, the information encoded across PPC layers during refined sensorimotor behavior remains largely unknown. To uncover the sensorimotor features represented in PPC during voluntary whisking and object touch, we performed loose-patch single-unit recordings and extracellular recordings of ensemble activity, covering all layers of PPC in anesthetized and awake, behaving male rats. First, using single-cell receptive field mapping, we revealed the presence of coarse somatotopy along the mediolateral axis in PPC. Second, we found that spiking activity was modulated during exploratory whisking in layers 2-4 and layer 6, but not in layer 5 of awake, behaving rats. Population spiking activity preceded actual movement, and whisker trajectory endpoints could be decoded by population spiking, suggesting that PPC is involved in movement planning. Finally, population spiking activity further increased in response to active whisker touch but only in PPC layers 2-4. Thus, we find layer-specific processing, which emphasizes the computational role of PPC during whisker sensorimotor behavior.

Key words: cortical layers; motor planning; posterior parietal cortex; tactile coding; whisker somatotopy

\section{Significance Statement}

The posterior parietal cortex (PPC) is thought to merge information on motor output and sensory input to orchestrate interaction with the environment, but the function of different PPC microcircuit components is poorly understood. We recorded neuronal activity in rat PPC during sensorimotor behavior involving motor and sensory pathways. We uncovered that PPC layers have dedicated function: motor and sensory information is merged in layers 2- 4; layer 6 predominantly represents motor information. Collectively, PPC activity predicts future motor output, thus entailing a motor plan. Our results are important for understanding how PPC computationally processes motor output and sensory input. This understanding may facilitate decoding of brain activity when using brain-machine interfaces to overcome loss of function after, for instance, spinal cord injury.

\section{Introduction}

The posterior parietal cortex (PPC) is anatomically located between primary somatosensory ( $\mathrm{S} 1$ ) and visual (V1) cortices and is intricately connected to multiple cortical, thalamic, and other subcortical regions (Wang et al., 2012; Wilber et al., 2014). The

\footnotetext{
Received March 3, 2019; revised June 24, 2019; accepted July 7, 2019.

Author contributions: H.M., J.F.S., and C.P.J.d.K. designed research; H.M., R.B., A.W.P., and R.d.H. performed research; H.M., R.d.H., R.B., A.W.P., and C.P.J.d.K. analyzed data; H.M. wrote the first draft of the paper; H.M., R.d.H., F.H., J.F.S., H.D.M., and C.P.J.d.K. edited the paper; H.M. and C.P.J.d.K. wrote the paper.

This work was supported by the Center for Neurogenomics and Cognitive Research, Amsterdam Neuroscience, and VU Amsterdam NWO-ALW OPEN 822.02.013 to C.P.J.d.K., and ENC-Network p3-c3 Grant to C.P.J.d.K., F.H., and J.F.S. We thank Lynnet Frijlling for assistance with Nissl stainings; and Eline Mertens for Neurolucida reconstructions. The authors declare no competing financial interests.

Correspondence should be addressed to Christiaan P.J. de Kock at ckock@falw.vu.nl.

H. Mohan's present address: Cold Spring Harbor Laboratory, 1 Bungtown Road, Cold Spring Harbor, NY 11724
}

PPC has consequently been implicated in a broad spectrum of sensory, motor, and cognitive behaviors, including sensorimotor processing, decision making, spatial navigation, attention, route planning/reaching, and multisensory integration (Chen et al., 1994; Andersen et al., 1997; Bucci, 2009; Carandini and Churchland, 2013; Whitlock, 2014, 2017; Goard et al., 2016; Krumin et al., 2018; Mimica et al., 2018; Mohan et al., 2018; Nikbakht et al., 2018). The contribution of PPC to many different behaviors complicates efforts to disentangle its functional architecture and

R. Broersen's present address: Eccles Institute of Neuroscience and Australian Research Council Centre of Excellence for Integrative Brain Function, John Curtin School of Medical Research, Australian National University, Canberra 0200, ACT, Australia.

https://doi.org/10.1523/JNEUROSCl.0693-19.2019

Copyright $(2019$ the authors 
the coding principles that underlie different behaviors. One approach to uncover which features of sensorimotor behavior are encoded in rodent PPC is to simplify task parameters and study neuronal function in awake, behaving animals with tight stimulus control, which can be achieved, for example, during sensorimotor behavior, including voluntary whisking and object touch in head-fixed rodents.

Rodents, such as rats and mice, actively use their whiskers to construct a sensory percept of their environment, and sensorimotor transformations are fundamental to a broad repertoire of cognitive behaviors. During active exploration of the environment, whisker movements continue to be under motor control, and correct integration of motor and sensory signals is thus crucial for appropriate behavioral performance. The neuronal circuitry orchestrating whisker-guided sensorimotor behavior consists of well-defined pathways connecting sensory and motor areas (Petersen, 2007; Feldmeyer et al., 2013). Rodent PPC is reciprocally connected to whisker somatosensory and motor cortices, and PPC thus anatomically bridges incoming sensory information from the whiskers and outgoing motor commands to potentially orchestrate whisker motion (summarized in Mohan et al., 2018). Since whisker-guided behavior is so fundamental to rodent behavior, understanding the functional architecture of rodent PPC during innate whisker-based somatosensation may facilitate understanding the cortical coding principles during increased cognitive load, for instance, during learned whiskerguided stimulus detection (e.g., Le Merre et al., 2018) or twoalternative forced choice performance (e.g., Guo et al., 2014).

The involvement of rodent PPC during somatosensory processing is supported by evidence of haptic processing after passive or active tactile stimulation (McNaughton et al., 1994; Olcese et al., 2013; Nikbakht et al., 2018). On the other hand, mice performing a whisker-based decision-task will continue to perform accurately after neuronal activity in PPC is silenced (Guo et al., 2014; Le Merre et al., 2018), challenging the necessity of PPC in bridging motor and sensory pathways during tactile perception. It remains an open question, therefore, if and how PPC is activated during voluntary whisker-based somatosensation, and which specific features of tactile behavior are represented in PPC of awake, behaving rodents.

To fill this gap, we used single-cell, loose-patch and multielectrode recordings in both urethane-anesthetized and awake, behaving rats and found a highly organized coding scheme in PPC where specific features of sensory and motor information are represented across specific layers, which may support a broad spectrum of whisker-guided behaviors.

\section{Materials and Methods}

Anesthetized animal preparation. This study was performed in accordance with European and Dutch law and approved by the animal ethical care committee of the VU Amsterdam and VU University Medical Center. Urethane-anesthetized $(1.6-1.7 \mathrm{~g} / \mathrm{kg}$ ) male Wistar rats (Harlan, $n=$ 38 , average \pm SD: postnatal day $35 \pm 5$, body weight $125 \pm 43 \mathrm{~g}$ ) were used. Depth of anesthesia was checked by foot and eyelid reflex. The animal's temperature was monitored and maintained at $37^{\circ} \mathrm{C}$ using a rectal probe and a thermostatically controlled heating pad during surgery and experiment. For passive whisker stimulation, all whiskers contralateral to the recorded (left) hemisphere were trimmed to $5 \mathrm{~mm}$ relative to whisker follicle. Single whiskers were subsequently deflected in random order by a glass capillary attached to a piezoelectric bimorph. The posterior edge of primary somatosensory cortex (S1) was identified using intrinsic optical imaging through deflection of individual straddler whiskers $(\gamma, \delta)$. The cortical strip posterior to the straddlers was targeted as the recording site (centered at $3.5 \mathrm{~mm}$ posterior and $4.5 \mathrm{~mm}$ lateral from bregma and confined within the medial and lateral boundaries of S1).

Loose-patch recording and receptive field $(R F)$ mapping. In vivo loosepatch recordings were made as previously described (de Kock et al., 2007). Briefly, borosilicate filamented glass pipettes with $5-8 \mathrm{M} \Omega$ resistance and filled with the following (in $\mathrm{mM}$ ): $135 \mathrm{NaCl}, 5.4 \mathrm{KCl}, 1.8 \mathrm{CaCl}_{2}$, $1 \mathrm{MgCl}_{2}$, and 5 HEPES were used to record from individual neurons across the cortical depth of PPC. Pipette solution was supplemented with $20 \mathrm{mg} / \mathrm{ml}$ biocytin to allow extracellular deposits (Moore et al., 2015a) or dye-loading of recorded neurons for post hoc staining, to determine its position with respect to the barrel cortex anatomical landmarks and for reconstruction of single-cell morphologies (Pinault, 1996; de Kock, 2016). Cell search was performed while monitoring electrode resistance to record from an unbiased sample of PPC neurons, independent of spiking frequencies of individual neurons.

Histology and reconstruction. The histology procedure used to reveal anatomical landmarks and recorded neurons has previously been described (Wong-Riley, 1979; Horikawa and Armstrong, 1988; Narayanan

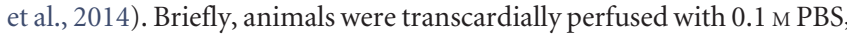
$\mathrm{pH}$ 7.2, followed by $4 \%$ PFA, and brains were removed and fixed in PFA for $24 \mathrm{~h}$. Twenty-four $100-\mu \mathrm{m}$-thick tangential sections were obtained, and cytochrome oxidase staining was used on sections $6-11$ to reveal anatomical landmarks in primary somatosensory cortex (S1). The chromagen 3,3' diaminobenzidine tetrahydrochloride was used for staining to reveal dendritic architecture and position of recorded neurons with respect to S1. For a subset of recordings, barrel architecture, slice boundaries, and cell location were reconstructed in 3D under a brightfield microscope, using Neurolucida software (Microbrightfield) (de Kock et al., 2007). Silicon probes were coated with $1,1^{\prime}$-dioctadecyl 3,3,3',3'tetramethylindocarbocyanine perchlorate to label the insertion spot. After recordings and perfusion, cytochrome oxidase staining was performed and tangential sections viewed using fluorescence microscopy to confirm PPC recording location.

Nissl stainings were performed on four brains to obtain landmarks for cytoarchitecturally defined layers. Following PFA fixation, $100-\mu \mathrm{m}-$ thick coronal sections were stained with $0.5 \%$ cresyl violet and imaged under a brightfield microscope. Cell density was measured by obtaining average pixel intensity across a rectangular strip of the PPC ranging from pia to white matter. We consistently found a change in pixel brightness at $850-1250 \mu \mathrm{m}$ depth (see Fig. $2 A$ ), resulting in identification of three compartments. Increased contrast was associated with increased soma size, implying that the middle compartment corresponds to layer 5 (Chagnac-Amitai et al., 1990; Defelipe, 2011; Tomassy et al., 2014; SiglGlöckner and Brecht, 2017). Putative identity of L5 was confirmed by single-cell morphologies of representative examples (see Fig. $2 A$ ). We thus define three borders that separate cytoarchitectural compartments: $\mathrm{L} 1 / 2$ border at $208 \pm 35 \mu \mathrm{m}, \mathrm{L} 4 / 5$ border at $856 \pm 75 \mu \mathrm{m}$, and L5/6 border at $1242 \pm 47 \mu \mathrm{m}$. Electrophysiological recordings were categorized based on recording depth and classified as L2-4, L5, and L6.

Data analysis of loose-patch recordings in anesthetized animals. Recordings were made using an Axoclamp 2B amplifier (Axon Instruments), in combination with a Lynx 8 amplifier filtered between 300 and $9000 \mathrm{~Hz}$. We used custom-made software on Labview (National Instruments) to acquire data (nTrode, Randy Bruno, Columbia University, New York). Spikes were sorted offline using Mclust 2.0 (A. David Redish, University of Minnesota, Minneapolis).

Spontaneous activity was recorded for $100 \mathrm{~s}$ continuously or using the $100 \mathrm{~ms}$ prestimulus episode during whisker stimulation over repeated trials for each neuron. Evoked activity was quantified by deflection of single whiskers using a piezoelectric bimorph attached to a glass capillary. Individual whiskers were deflected $20 \times$ in the rostrocaudal direction at 3.3 degrees with a rise time of $8 \mathrm{~ms}$, an onset-offset interval of $200 \mathrm{~ms}$, and an intertrial interval of $2000 \mathrm{~ms}$. Evoked activity was determined in the $0-200 \mathrm{~ms}$ poststimulus time window and corrected for spontaneous activity to compute the number of spikes per stimulus (see Fig. $2 G-I$ ).

The subthreshold local field potential (LFP) maps were constructed by quantifying the average of the integral of $4 \mathrm{~Hz}$-high pass filtered, stimulus-evoked LFP in the $0-100 \mathrm{~ms}$ poststimulus window after subsequent deflection $(20 \times)$ of individual whiskers. The integral was baseline- 
corrected using the $0-100 \mathrm{~ms}$ prestimulus window. Recording locations were color-coded corresponding to the row associated with the whisker generating the maximum LFP response (see Fig. 1D).

Awake animal preparation. Male Wistar rats (Harlan, $n=12$, mean \pm SD: postnatal day $34 \pm 5$, body weight $128 \pm 27 \mathrm{~g}$ ) were used for loosepatch single-cell $(n=9)$ and silicon probe multielectrode $(n=3)$ recordings during free whisking and object touch. During surgery, animals were anesthetized with $1.5 \%$ isoflurane in $0.4 \mathrm{l} / \mathrm{h} \mathrm{O}_{2}$ and $0.7 \mathrm{l} / \mathrm{h} \mathrm{NO}_{2}$. The skull over the PPC in the left hemisphere was thinned at $3.5 \mathrm{~mm}$ posterior to bregma and $4.5 \mathrm{~mm}$ lateral from midline and protected using a plastic cylinder and screw cap. A metal headpost was firmly attached to the skull using dental cement (Tetric EvoFlow, Ivoclar Vivadent) after which rats were allowed to recover for $24 \mathrm{~h}$ (Boudewijns et al., 2013). Animals were habituated to head fixation by daily training with increasing duration (5-25 min). Rats were housed in enriched cages (shelter, bedding material, toys) with ad libitum food and water, and body weight was monitored throughout the entire habituation period. Recording sessions were initiated when rats were habituated to head fixation.

Recording in awake cortex. On the recording day, the rat was anesthetized with $1.5 \%$ isoflurane $\left(0.4 \mathrm{l} / \mathrm{h} \mathrm{O}_{2}\right.$ and $\left.0.7 \mathrm{l} / \mathrm{h} \mathrm{NO}_{2}\right)$ and a craniotomy performed over the thinned region of interest. Borosilicate glass pipettes were used to record from individual neurons. While recording, whiskers were individually deflected using a glass capillary attached to a bimorph piezo, to identify the whisker row generating strongest LFP activity. Either a single or the three most caudal whiskers of the preferred row were spared. Spontaneous activity was recorded for $100 \mathrm{~s}$ followed by extracellular current injection to facilitate dye-loading of biocytin (Pinault, 1996; Narayanan et al., 2014). Afterward, neurons were allowed to recover from the filling procedure until prefilling spiking conditions were reestablished (Herfst et al., 2012). Anesthesia was then stopped, after which rats quickly recovered (Kortelainen et al., 2012; Boudewijns et al., 2013). Whisking and touch behavior was recorded at 200 or $400 \mathrm{~Hz}$ using a Nikon AF Nikkor $50 \mathrm{~mm}$ f/1.4D camera under infrared light illumination. Whisker position was tracked offline using MATLAB-based software WhiskerTracker (The MathWorks) (Knutsen et al., 2005) or python-based "whisk" (Clack et al., 2012). The object (hexagon key, diameter $1.5 \mathrm{~mm}$ ) was positioned $\sim 2 \mathrm{~cm}$ lateral from the whisker pad and anterior relative to the whisker setpoint (obtained during quiescent episodes). This ensured that touches were the consequence of whisker protraction. Additionally, the proximal position with respect to the whisker follicle ensured that rats would not generate "slip-of" events, which can occur with distal object positions (Hires et al., 2013). Touch start and end times were extracted by manual inspection of video files frame by frame.

Extracellular spiking activity was recorded using probes (E32-50-S1L6, Atlas Neuroengineering) with 32 iridium oxide electrodes bearing a pitch of $50 \mu \mathrm{m}$ and spanning $1550 \mu \mathrm{m}$. Probes coated with $1,1^{\prime}$ dioctadecyl 3,3,3',3'-tetramethylindocarbocyanine perchlorate were inserted to a depth of $1900 \mu \mathrm{m}$ from the pia and extracellular spiking activity acquired at $30 \mathrm{kHz}$ using open ephys acquisition board and recorded using open ephys GUI software (http://www.open-ephys.org/ gui/). The signal was bandpass-filtered $(0.3-6 \mathrm{kHz})$ before offline sorting.

Spike sorting. Spike sorting for loose-patch recordings of single cells was performed in Mclust 2.0 through manual clustering. Spike sorting for multielectrode recordings was performed in Mclust 4.3 (Fraley and Raftery, 2002, 2003) with units first clustered semiautomatically using klustakwik (Harris et al., 2000), subsequently assisted by manual curation. Spike sorting was performed based on previously described methods (Csicsvari et al., 1999; Harris et al., 2001, 2016; Barthó et al., 2004). For clustering, channels were grouped into tetrodes (i.e., four consecutive channels were used to extract spike waveforms online). Waveforms from each "tetrode" were further semiautomatically sorted using klustakwik to obtain isolation distance for all clusters. Isolated units were checked during the manual curation phase for various parameters, including spikes within a refractory period of $2 \mathrm{~ms}$, spike shapes, auto- and cross-correlograms, and stability of spiking activity throughout the full recording session. Only clusters that were identified as well isolated were used for further analyses. Clusters on neighboring tetrodes, identified using cross-correlations of their spike times, were only processed once and discarded from the adjacent tetrode group to prevent multiple copies of the same unit.

Cell type categorization based on spike waveform was performed after extracting action potential (AP) half-width and peak-to-trough latency from the average waveform for each unit. Units were defined as fast spiking units (FSU) and regular spiking units (RSU) based on waveform shape identifying putative interneurons and putative pyramidal neurons (FSU peak-to-trough $<0.38 \mathrm{~ms}$, RSU peak-to-trough $>0.45 \mathrm{~ms}$ ) (Barthó et al., 2004).

Data analysis of population activity. A spiking activity map was generated for individual recording sessions (e.g., single trials of $250 \mathrm{~s}$ ) from a single rat by binning spikes at $50 \mathrm{~ms}$ resolution for all neurons recorded simultaneously across PPC layers $\left(\mathrm{S}_{\mathrm{n}, \mathrm{t}}\right.$, dimensions: number of units $(n)$ * number of time bins (t); see Fig. 4A1). The perievent time histogram $(\mathrm{PETH})$ was calculated for each unit based on its spiking rate centered to the onset of consecutive whisking events spanning $2 \mathrm{~s}$ before and after (20 ms bin size). Thus, single-trial analyses were performed at $50 \mathrm{~ms}$ resolution, multievent analyses at $20 \mathrm{~ms}$ resolution. $z$-scored activity from the PETH was calculated for each unit with respect to the baseline activity. $z$-scored activity for all units recorded simultaneously was stacked into a matrix to generate a $z$-scored activity map $\left(Z_{\mathrm{n}, \mathrm{tz}}\right.$, dimensions: number of units $(n){ }^{*}$ number of time bins (tz); see Fig. 4A2). Principal component (PC) analysis to obtain the first PC was performed on the $z$-scored activity map ranging from $200 \mathrm{~ms}$ before whisking onset to $1000 \mathrm{~ms}$ after whisking onset.

$$
\operatorname{PCA}\left(Z_{\mathrm{n}, \mathrm{tz}}^{\mathrm{T}}\right)=\mathrm{W}_{\mathrm{n}, \mathrm{n}}
$$

The projection (Proj; see Fig. 4A3) of spiking activity onto the first PC was obtained by the dot product between the single-trial spiking activity matrix $\left(\mathrm{S}_{\mathrm{n}, \mathrm{t}}\right)$ and the first PC $\left(\mathrm{w}_{\mathrm{n}, 1}\right)$ as follows:

$$
\operatorname{Proj}_{\mathrm{t}, 1}=\mathrm{S}_{\mathrm{n}, \mathrm{t}}^{\mathrm{T}} \cdot \vec{w}_{\mathrm{n}, 1}
$$

To obtain the whisking envelope, whisking $\theta$ was first bandpass-filtered between 2 and $20 \mathrm{~Hz}$. The filtered signal was then used to obtain the upper peak whisking envelope using spline interpolation over the local maxima separated by $250 \mathrm{~ms}$. Whisking envelope was then downsampled to match the length of the projection. Spiking projections were filtered with a $250 \mathrm{~ms}$ moving average filter before measuring correlation coefficient between whisking envelope and spiking projections. Pearson's linear correlation was used to obtain the correlation coefficient between the spiking projection activity and whisking envelope for each trial lasting $250 \mathrm{~s}$. To obtain shuffled correlations, spike times for each neuron were shuffled 1000 times for each rat and then analyzed as before. The neural network used to predict whisking trajectory consisted of 1 hidden layer with 5 neurons and used Bayesian regularization back propagation to optimize weights (Neural Network Toolbox, MATLAB 2017a, The MathWorks). The spiking projection from one $250 \mathrm{~s}$ trial was split into 75:25 training and cross-validation sets, and the neural network was subsequently trained on the training set and used to predict whisker projection endpoints in the test set.

Statistics. Instat 3 (GraphPad) or MATLAB 2017a (The MathWorks) was used for statistical analysis. To test somatotopy (see Fig. 1), we transformed single RFs into a matrix with whisker row on the $x$ axis $(\mathrm{A}=1$, $\mathrm{B}=2, \mathrm{C}=3, \mathrm{D}=4$, and $\mathrm{E}=5$ ) and maximum whisker response for each row on the $y$ axis. Next, a Spearman's correlation was performed for individual neurons between the row and maximum response to extract the correlation coefficient for individual neurons. Lateral neurons tuned to dorsal whisker stimulation should show a negative correlation coefficient; medial neurons tuned to ventral whiskers should show a positive correlation coefficient. To determine somatotopy in PPC, we finally performed a Pearson's correlation between the anatomical coordinate along the mediolateral axis versus single-cell correlation coefficients.

In Figures 2-5, data were generally not normally distributed. Therefore, we used the nonparametric ANOVA (Kruskal-Wallis test) for comparison between layers $2-4,5$, and 6 , followed by Dunn's post hoc test for comparison of two layers (see Fig. $2 F, I$ ) or Friedman Test (nonparametric repeated-measures ANOVA) with post hoc tests to test the effect of 
A

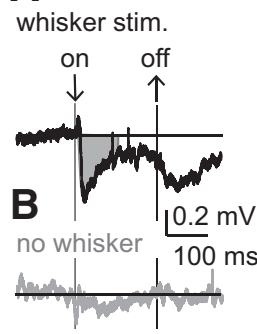

C

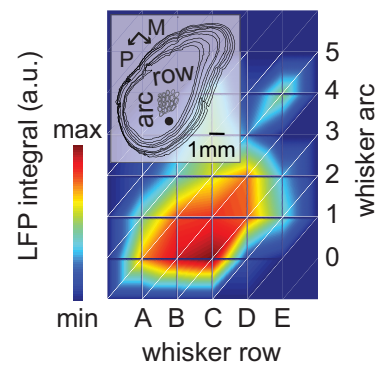

D

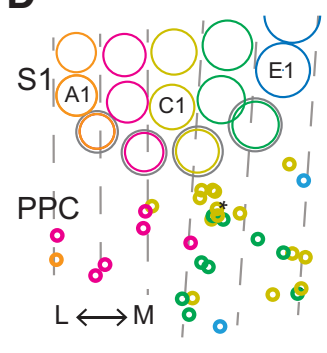

$E$

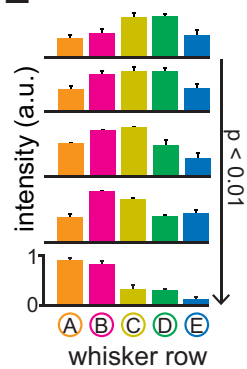

Figure 1. LFP RF mapping reveals coarse functional somatotopy in PPC. $\boldsymbol{A}$, Example recording represents the average LFP after 20 consecutive single whisker deflections. The integral (gray shading) of the $0-100$ poststimulus window is used to quantify the single-whisker LFP integral. Arrows indicate onset and offset for whisker deflection. $\boldsymbol{B}$, Control condition with piezo in place but without whisker contact to illustrate the tactile nature of LFP in PPC after whisker deflection. C, Example LFP RF heat map for an individual PPC neuron showing LFP integral across individual whiskers, normalized to the maximal integral value. The preferred single whisker was used to color code the recording site; here $\gamma$ (Fig. 1D, asterisk). Inset, Digital reconstruction of serial tangential sections together with S1 landmarks (barrels in L4) and position of recorded neuron from the example in A-C. D, All recording locations projected onto a standardized reference frame. Color code of recording location matches the color code of the whisker that generated the maximum response. $\boldsymbol{E}$, From top to bottom: medial to lateral PPC subregions. Bar plots represent the normalized mean LFP integral per whisker row for the five PPC subregions, delineated by extrapolation of barrel row organization ( $n=33$ recordings from $N=29$ rats). Coarse somatotopy can be inferred from a gradual shift from dorsal (A-row) to ventral (E-row) whiskers along the mediolateral axis. P: posterior, M: medial, L: lateral.

whisking or touch on single-unit spiking rates (see Figs. 3F2, 5G). Significance level was set at $p<0.05$.

\section{Results}

\section{Somatotopic functional organization in PPC}

$\mathrm{PPC}$ receives direct input from primary somatosensory (barrel) cortex through somatotopically aligned projections (Lee et al., 2011), but it has not been studied how this translates into functional somatotopy in PPC. To determine whether functional somatotopy can be uncovered in PPC, we quantified LFPs carrying unimodal tactile information using loose-patch recordings in urethane-anesthetized rats (Fig. $1 A-E$ ). Whisker-evoked LFPs were quantified as the integral of the loose-patch recorded field potential in the $0-100 \mathrm{~ms}$ poststimulus window (Fig. $1 A$ ). The RF was obtained by subsequently deflecting individual whiskers and computing the whisker-specific response integrals (Fig. 1C; see Materials and Methods). Neurons were biocytin-loaded (or an extracellular deposit used) and anatomical landmarks reconstructed to annotate RFs to a standardized anatomical framework (Fig. 1C, inset) (Egger et al., 2012). The preferred (or principal) whisker was obtained by identifying the whisker evoking the maximum LFP integral. The color code associated with the recording location corresponds to the row to which the preferred whisker belongs (Fig. 1D; see Materials and Methods). Barrel centers were used to spatially delineate areas of PPC corresponding to specific whisker barrel rows, and average tuning values of individual neurons to whisker rows were determined for these 5 subregions of PPC (Fig. 1E). We found that the preferred whisker representation showed a significant shift from dorsal whiskers (A-row) in lateral recording positions to ventral whiskers when recording from medial locations (Fig. $1 E ; n=29$ rats, $r=0.6, p<$ 0.01 , Pearson correlation; see Materials and Methods). Thus, input (LFP) maps of PPC neurons show functional somatotopy, albeit at coarse spatial resolution.

\section{Spontaneous and evoked activity across PPC layers}

We performed loose-patch recordings of individual neurons across PPC layers of urethane-anesthetized rats to characterize suprathreshold processing of whisker somatosensory information in PPC. Post hoc histology was performed to determine layer and recording location with respect to S1 border (Fig. 2A,B). Single-cell morphology and/or recording depth was used to assign individual units to layer $2-4(200-850 \mu \mathrm{m}$ from pia), L5
$(850-1250 \mu \mathrm{m})$, or L6 $(1250-1850 \mu \mathrm{m})$ (for delineation of borders, see Materials and Methods). Only units with regular spike waveform (peak to trough $>0.45 \mathrm{~ms}$, putative pyramidal neurons) were included in analyses (Barthó et al., 2004). Spontaneous and evoked spiking activity was acquired to determine suprathreshold processing of whisker somatosensory information in PPC (Fig. 2C,D). We found that spontaneous activity was depth-dependent and significantly higher in L5 compared with L2-4 (Fig. 2E, F; Table 1: median values, first through third quartiles).

To determine layer-specific whisker-evoked activity in PPC, we quantified AP spiking in response to passive whisker deflection (Fig. 2G-I). Individual whiskers were deflected 20 times and single-cell raster plots constructed (data not shown). The whisker with the maximum response was identified as the principal whisker (Fig. 2G), and evoked activity was corrected for baseline (spontaneous) activity. Similar to spontaneous activity, we found that evoked responses were depth-dependent and significantly higher in L5 compared with L2-4 (Fig. 2 H,I; Table 1).

We almost exclusively found multiwhisker RFs in PPC recordings, indicating that evoked activity could be elicited by multiple whiskers ( $n=18$, data not shown), which is in line with broad LFP RFs and coarse somatotopy. To conclude, spontaneous and whisker-evoked spiking activity during urethane anesthesia is layer-specific and significantly higher in L5 compared with L2-4.

Layer-specific encoding of voluntary whisker motion in PPC To characterize encoding of exploratory whisking in PPC, we recorded single-unit spiking activity in awake rats across all PPC layers simultaneously using (32-channel) laminar silicon probes (Fig. 3A,B; see Materials and Methods). Average spike waveforms for individual units were used to separate RSUs (putative excitatory neurons, $n=108$ ) from FSUs (putative inhibitory, $n=$ 11; Fig. 3C; see Materials and Methods). High-speed videography $(400 \mathrm{~Hz})$ was used for offline whisker tracking, and behavior was categorized into episodes of quiescence (Q) or free whisking (FW) using custom software (Movie 1).

Spiking frequency of individual units changed upon FW (Fig. $3 D$; Table 2), and the change was specific for the RSU population and layer-dependent (Fig. 3E, F1,F2). More specifically, spiking frequency significantly increased in L2-4 and L6 but not in L5 
A

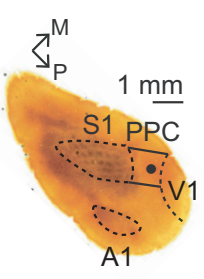

B

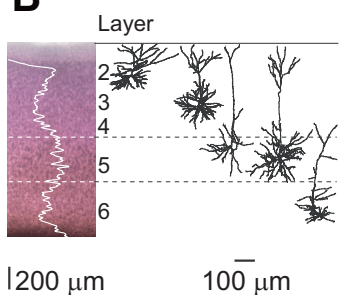

C

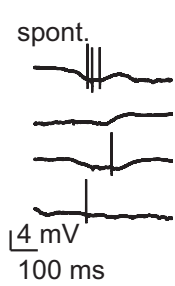

G

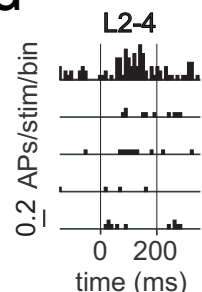

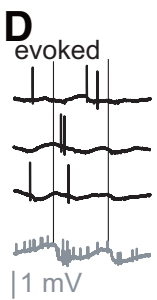
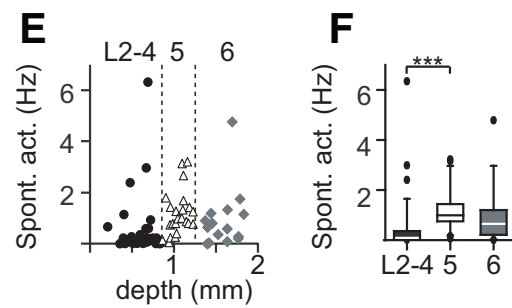

H

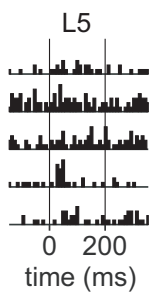

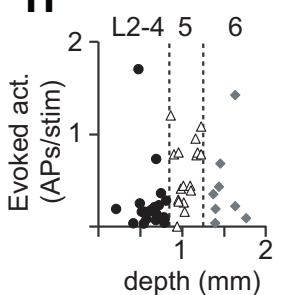

I

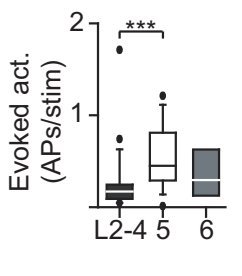

Figure 2. Layer-specific processing of passive tactile stimulation. $A$, Tangential section of neocortex after cytochrome oxidase staining to reveal L4 of primary somatosensory (S1), primary visual (V1), and primary auditory cortex (A1) to localize recorded neuron. B, Coronal section of PPC after Nissl staining to reveal layers. White line (superimposed) indicates pixel contrast. Increased pixel contrast at $\pm 850-1250 \mu \mathrm{m}$, in combination with cell-body size and single neuron morphologies was used to delineate $L 5$ and adjacent compartments (L2-4, L6). C, Example loose-patch recording with snapshots of spontaneous activity of a single PPC neuron. D, Example recording illustrating spiking activity during 3 consecutive whisker-stimulation trials (Trials 3-5). Gray trace represents the average of all 20 single whisker stimulation trials. Thin vertical lines indicate onset and offset of whisker stimulation, respectively. E, Spontaneous spiking activity (in $\mathrm{Hz}$ ) of individual neurons across PPC layers. Dashed lines indicate the border between L2 - 4, L5, and L6.F, Spontaneous activity is significantly higher in L5 compared with L2 4 (Kruskal-Wallis with Dunn's Multiple Comparisons Test, $p<0.001 ; \mathrm{L} 2-4, n=35 ; \mathrm{L} 5, n=23 ; \mathrm{L} 6, n=15$ ). $\mathbf{G}$, Example peristimulus-time histograms for a subset of individual units from $\mathrm{L} 2-4$ and $L 5$. $\boldsymbol{H}$, Evoked activity (APs/stim) of individual neurons across PPC layers. Dashed lines indicate the border between L2-4, L5, and L6. I, Evoked activity (APs/stim) is significantly higher in L5 compared with L2-4 (Kruskal-Wallis with Dunn's Multiple Comparisons Test, $p<0.01 ; \mathrm{L} 2-4, n=22 ; \mathrm{L} 5, n=17 ; \mathrm{L} 6, n=8) .{ }^{* * *} p<0.001$.

Table 1. Layer-specific spiking activity in PPC of anaesthetized rats ${ }^{a}$

\begin{tabular}{lll}
\hline Layer & Spontaneous activity $(\mathrm{Hz})$ & Evoked activity (APs/stim) \\
\hline $2-4$ & $0.09(0.04-0.34), n=35$ & $0.17(0.10-0.24), n=22$ \\
5 & $0.99(0.77-1.44), n=23^{*}$ & $0.45(0.30-0.81), n=17^{*}$ \\
6 & $0.65(0.25-1.18), n=15$ & $0.30(0.18-0.50), n=8$ \\
\hline
\end{tabular}

${ }^{a}$ Values are median (first through third quartiles).

${ }^{*} p<0.001$.

(Table 2). The FSU population across layers did not change spiking frequency during free whisking (Table 2). Representation of self-induced exploratory whisking in PPC is thus layer- and cell type-specific and encoded by increased spiking frequency in RSUs in L2-4 and L6.

\section{Decoding whisker movement goals from population dynamics}

Exploratory whisking involves whisker trajectories that can cover a large parameter space (Towal and Hartmann, 2008), and protraction endpoints reflect intrinsically generated movement goals. To identify whether such movement goals are represented in rat PPC, we examined population dynamics at single-trial resolution during active somatosensation. Population spiking activity (Fig. 4A1) during a single recording trial was used to compute the $z$-scored heat map (Fig. 4A2), aligned to onset of whisking. Principal component analysis was then used on the $z$-scored activity to extract the variance in population spiking, reflecting the increase in spiking with the onset of whisking (Fig. 4A3). The single-trial spiking activity map (Fig. 4A1) was then (directly) projected onto the vector space to obtain the projection activity (Fig. 4A4). This projection activity thus embodies the variance in the single-trial population activity (Fig. 4A1). The whisker position recorded simultaneously was used to extract movement goals by generating an envelope over the local maxima of the whisker trajectory, reflecting the protraction endpoints (Fig. 4B, red dots). We consistently found a robust correlation between the whisking envelope and projection activity using temporally aligned projection activity and whisker envelope (Fig. $4 C 1 ; r_{\text {pro }}=$ $0.57, p<0.0001)$. Furthermore, correlation values significantly exceeded values obtained for data in which spiking times were shuffled (Fig. 4C2; $r=0.45$ ), and the projection of L2-4 and L6 populations was correlated more robust to whisker envelope relative to L5 populations. This is in line with our observation of layer-specific change in spiking activity during active somatosensation (Fig. 3). We also found significant correlations between ensemble activity and whisker envelope when we used coefficient of variation or mean spiking as alternative measures of population activity (rank-sum, $p<0.0001, r_{\text {mean }}=0.28, r_{\text {cov }}=0.22$, analyses not shown).

Next, we tested whether the cross-correlation between PCbased projection activity and whisker trajectory was maintained in the data when using the PC of one recording trial for the computation of projected activity during a subsequent but individual trial. This cross-projected activity was also robustly correlated to the corresponding whisking envelope (Fig. 4D1; $r=0.53$, $p<0.0001)$. We compared these correlation coefficients (cc) obtained from multiple rats $(n=3)$ against values obtained from shuffled spike times, first by combing spiking activity from all layers and then by extracting projections from units within each layer (Fig. 4D2; $r=0.41$ ). Also for cross-projected data, we found that spiking activity was significantly correlated to whisking envelope compared with shuffled spiking activity (Fig. 4D2), and projection activity from L2-4 and L6 populations was more strongly correlated to whisker envelope compared with the L5 population (Fig. 4D2). This indicates that the PC obtained during a single trial is sufficiently reliable across trials for the computation of correlations between ongoing population activity and whisker movements.

We ultimately determined whether projection activity from a single-trial PC was sufficient to predict movement goals during whisking. We trained a shallow neural network (Neural Network 
A

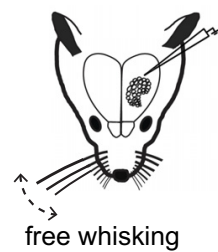

B

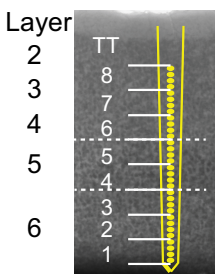

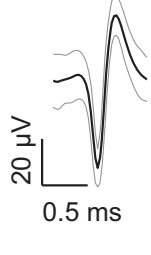

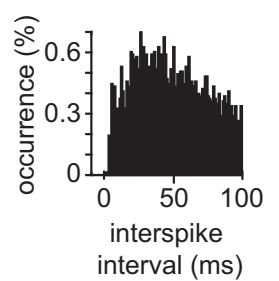

F1
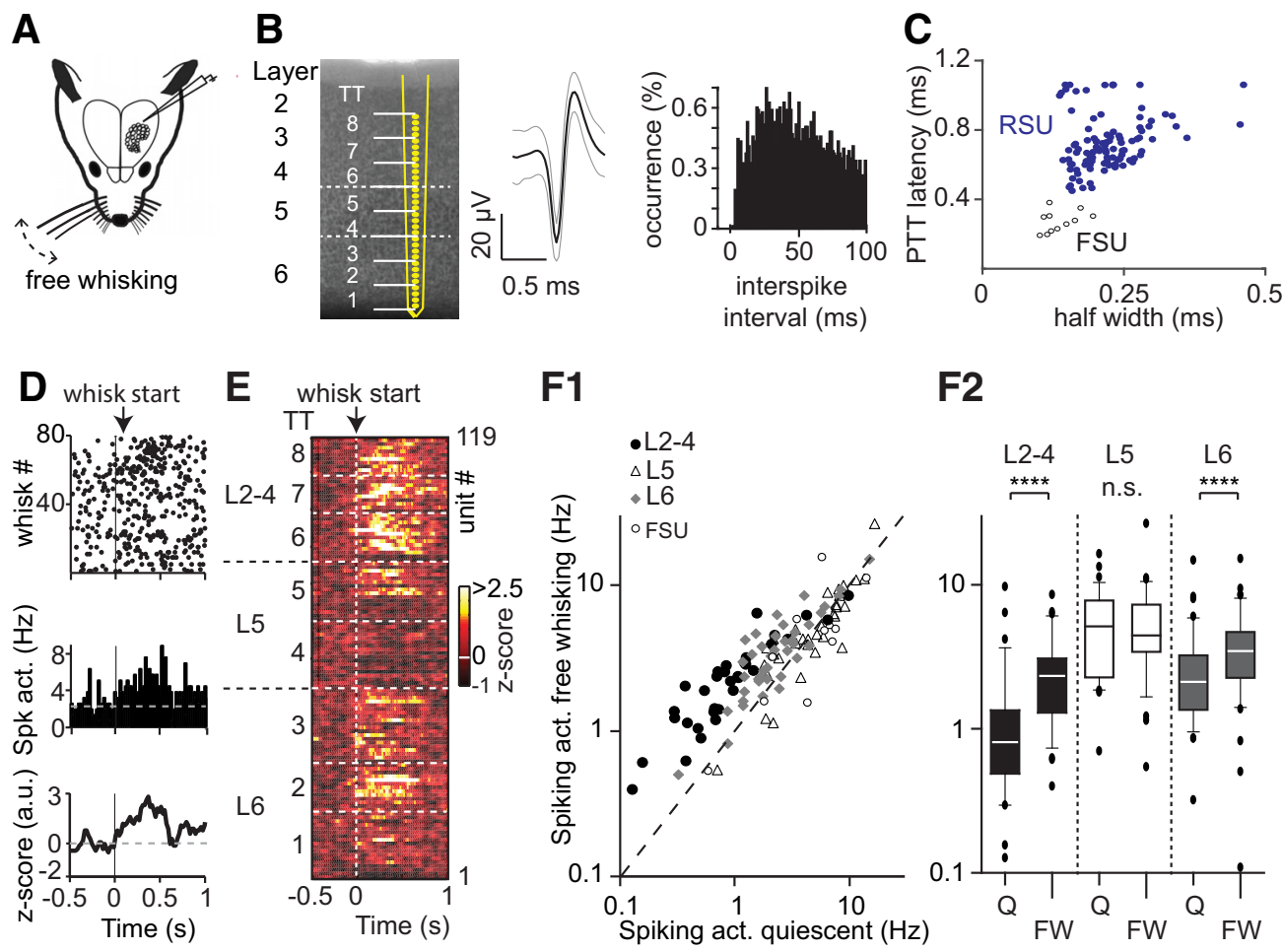

F2

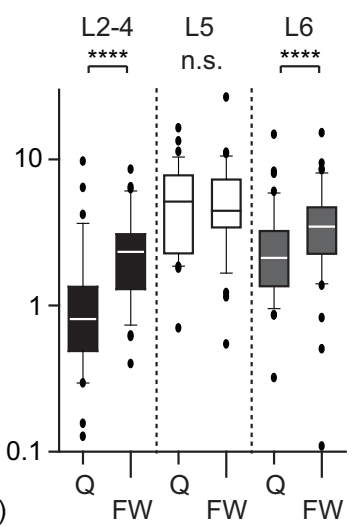

Figure 3. Layer-specific representation of free whisking in PPC. A, Experimental design for silicon probe recordings across PPC layers to quantify modulation of spiking activity upon free whisking (FW). B, Left, Structural layout of the 32-channel silicon probe across PPC layers. White dashed lines indicate borders between L2-4, L5, and L6. Middle, Single-unit waveforms with mean (black solid) and SEM (gray) superimposed. Right, Histogram of interspike intervals for same unit. C, Scatterplot of AP half-width and peak-to-trough latency for individual clusters across extracellular recordings. Filled blue bullets represent RSUs (putative excitatory units, $n=108$ ). Open bullets represent FSUs (putative inhibitory units, $n=11$ ). $\boldsymbol{D}$, Raster plot (top), PETH (middle), and $z$-transformed PETH (bottom) of an example unit from L2 - 4 aligned to FW onset. There is increased spiking activity during FW. E, Heat map of z-transformed spiking activity of all RSUs across PPC layers aligned to FW onset. Each row represents a single unit, arranged according to recording depth. White dashed lines indicate borders between tetrode groups. F1, Scatterplot with spiking activity during quiescent episodes ( $\mathrm{Hz}$ ) versus spiking activity during FW behavior (in $\mathrm{Hz}$ ). F2, Box plots illustrating population statistics for spike modulation across PPC layers during FW. Spiking activity is significantly increased in L2 -4 and $L 6$ (Wilcoxon signed-ranks test, $p<0.0001$ for both) but not in $L 5$ ( $p>0.05$ ). $n=33$ for L2 $-4, n=33$ for $L 5$, and $n=42$ for $L 6$. ${ }^{* * *} p<0.0001$.

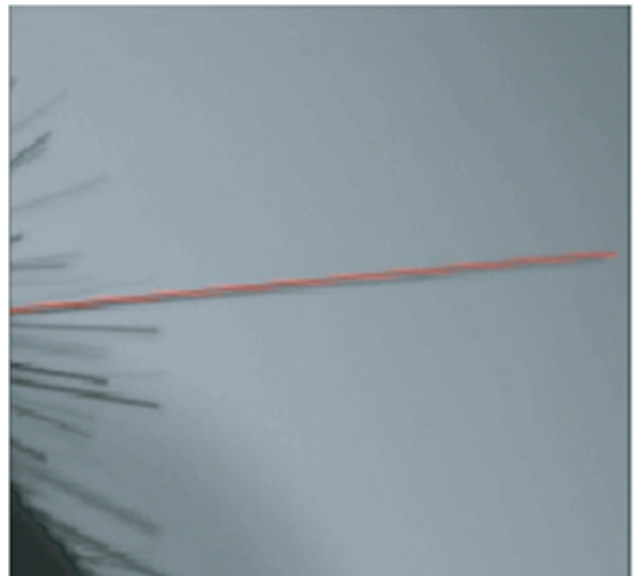

Movie 1. Free whisking video. Video shows a free whisking episode with (in red) the position of the offline tracked single whisker.

Toolbox, MATLAB 2014a, The MathWorks) with one hidden layer using the projection activity from one trial as the predictor and corresponding movement goals as the response variable. Using this decoder, we used cross-projection activity from a dif-
Table 2. PPC spiking during free whisking is layer- and cell type-specific ${ }^{a}$

\begin{tabular}{lll}
\hline Layer & Quiescent $(\mathrm{Hz})$ & Free whisking $(\mathrm{Hz})$ \\
\hline $2-4(n=33)$ & $0.81(0.50-1.25)$ & $2.33(1.36-2.95)^{*}$ \\
$5(n=33)$ & $5.14(2.39-7.61)$ & $4.45(3.47-7.19)$, NS \\
$6(n=42)$ & $2.12(1.43-3.19)$ & $3.46(2.28-4.63)^{*}$ \\
FSU $(n=11)$ & $4.24(1.94-6.48)$ & $4.13(1.59-5.47)$, NS
\end{tabular}

${ }^{a}$ Values are median (first through third quartiles).

${ }^{*} p<0.0001$; Quiescent versus Free whisking (Wilcoxon signed-ranks test).

ferent trial to predict movement goals, which correlated significantly with actual movements $(r=0.47, p<0.0001$; Fig. 4E1). Across trials, we found this correlation to be significantly larger than predictions made with shuffled spiking activity, and populations in L2-4 and L6 were able to predict movement goals that correlated strongly with actual movements compared with L5 populations (Fig. 4E2; $r=0.47$ ). To check whether spiking activity preceded protraction endpoints, we measured the variability of cross-correlations values between projection activity and corresponding whisker movement goals across temporal shifts and found that, in almost all trials, maximal correlation was found when projection activity preceded movement end goals (mean lead time $=109.1 \pm 89$ ms; Fig. $4 F ; p<0.01$ ). These results together suggest that whisker movement goals are reliably encoded by PPC neurons in a layer-specific manner. Finally, we also found modulation (albeit small) of population activity already before movement onset, reflecting increased spiking from a small subset of units before whisking onset (Fig. 4A3). Population ac- 
A1

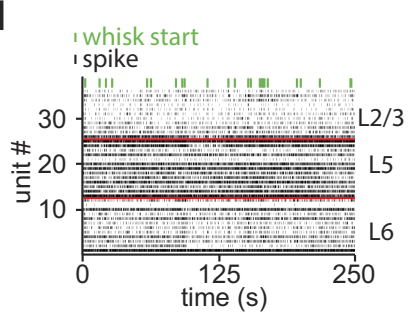

A2

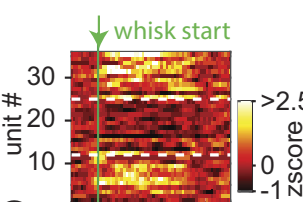

A3

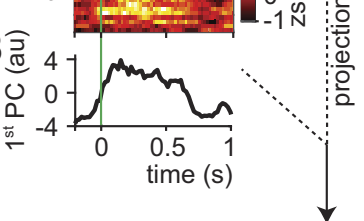

A4

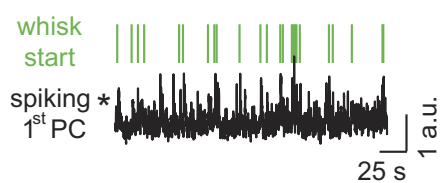

B

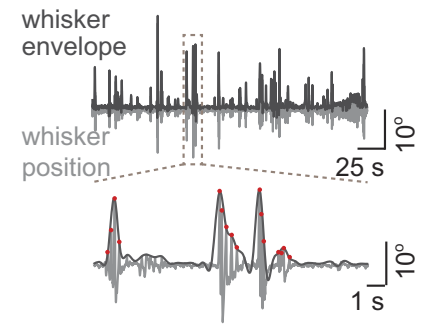

C1

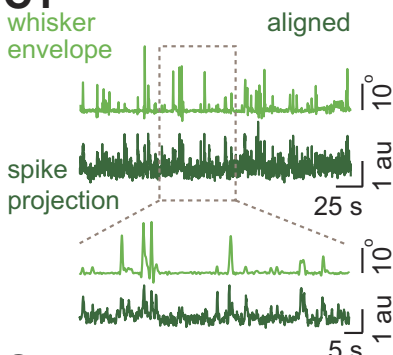

C2

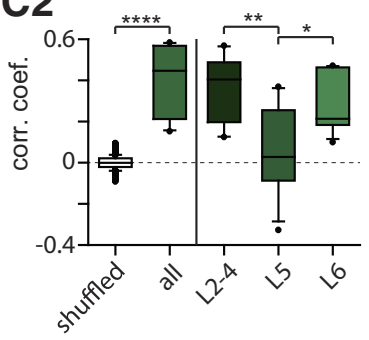

E1

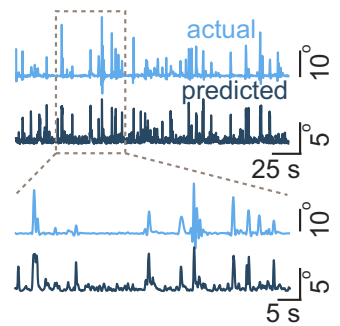

E2

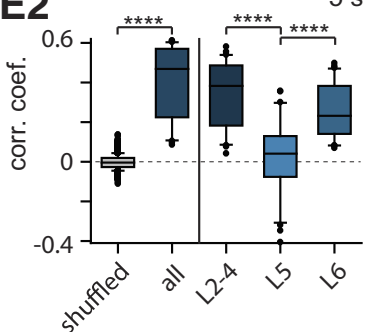

D1

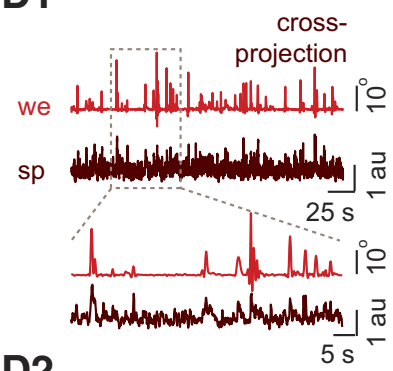

D2
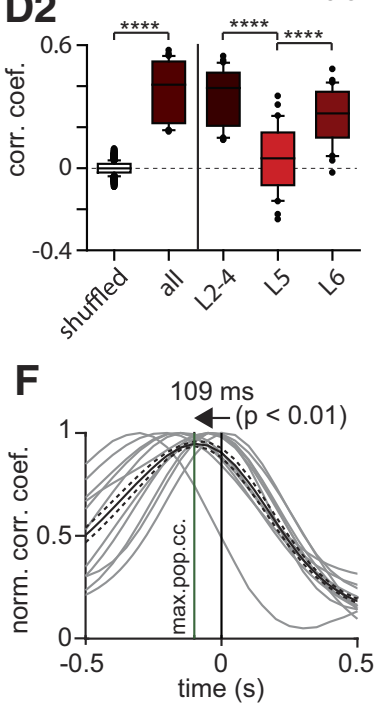

Figure 4. Decoding whisker movement goals from PPC population activity. A1, Single-trial spiking activity map of 36 units recorded simultaneously across PPC layers. Black ticks represent spikes binned at $50 \mathrm{~ms}$. Green ticks represent time stamps for onset of whisking. Red line indicates borders between L2-4, L5, and L6. A2, Heat map of the z-scored PETH of the 36 units (from A1) centered to onset of whisking (green line). A3, Projection of z-scored activity (from A2) onto its first PC. A4, Projection (black) of single-trial spiking activity map (from A1) onto the first PC of the z-scored activity (from $\mathbf{A 3}$ ). Green lines indicate time stamps for onset of whisking. $\boldsymbol{B}$, Top, Corresponding whisking $\theta$ (light gray) and its upper envelope (dark gray) indicate movement goals. Bottom, Zoomed in signal from a randomly selected temporal window. Red dots represent whisker projection endpoints (movement goals). C1, Top, Whisker envelope (light green) and corresponding direct-projection activity (dark green) obtained by projecting single-trial spiking activity onto the first PC (from z-scored activity) extracted from the same trial. Bottom, Zoomed in signal from a randomly selected temporal window. Note the correlation between movement goals and direct-projection activity. $\mathbf{C 2}$, Left, Correlation coefficient (cc) between whisker movement goals and direct-projection activity obtained from all units across layers compared against shuffled trials (Mann-Whitney $U$ test, $p<0.0001$ ). Right, cc between whisker movement goals and direct-projection activity obtained separately from units clustered within L2-4, L5, and L6 (one-way ANOVA with Tukey-Kramer post hoc test, $p<0.05$ ). D1, Top, Whisker envelope (light red) and corresponding cross-projection activity (dark red) obtained by projecting single-trial spiking activity onto the first PC (from z-scored activity) extracted from a different trial. Bottom, Zoomed in signal from a randomly selected temporal window. D2, Left, cc between whisker movement goals and cross-projection activity obtained from all units across layers compared against shuffled trials (MannWhitney $U$ test, $p<0.0001$ ). Right, cc between whisker movement goals and cross-projection activity obtained separately from units clustered within L2-4, L5, and L6 (one-way ANOVA with Tukey-Kramer post hoc test, $p<0.0001$ ). E1, Top, Actual whisker movement goals (light blue) and movement goals predicted from cross-projected activity (dark blue) using the neural network decoder. Bottom, Zoomed in signal from a randomly selected temporal window. E2, Left, cc between actual whisker movement goals and movement goals predicted from cross-projection activity obtained from all units across layers compared with shuffled trials (Mann-Whitney $U$ test, $p<0.0001$ ). Right, cc between actual whisker movement goals and movement goals predicted from cross-projection activity obtained separately from units clustered within L2-4, L5, and L6 (one-way ANOVA with Tukey-Kramer posthoc test, $p<0.0001$ ). $F$, Normalized cross-correlation between the direct-projection activity and whisker movement goals for each trial (light gray). Black represents average. Dashed line indicates SE. Green vertical line indicates average lead time (109.1 \pm 89 $\mathrm{ms}$, sign test, $p<0.01) .{ }^{*} p<0.05,{ }^{* *} p<0.01,{ }^{* * * *} p<0.0001$.

tivity can thus be used to reliably decode whisker movement, more precisely using spiking activity of L2-4 and L6 neurons. Since PPC activity predicts movement, our data strongly suggest that PPC is involved in motor planning of whisking.

\section{Layer-specific encoding of object touch}

We showed that PPC neurons encode information associated with intrinsically generated motor behavior, potentially repre- senting either efference copy (Cullen, 2004) or reafference signals (Cullen, 2004). To determine if and how ex-afferent whisker sensory information is represented, we performed loose-patch and silicon probe recordings across PPC layers during active object touch. Recording locations were confirmed by biocytin-loading individual neurons, or alternatively, dye labeling the recording site. Rats were habituated to head fixation, but otherwise naive to the sensory environment (Fig. 5A,B). We recorded AP spiking 

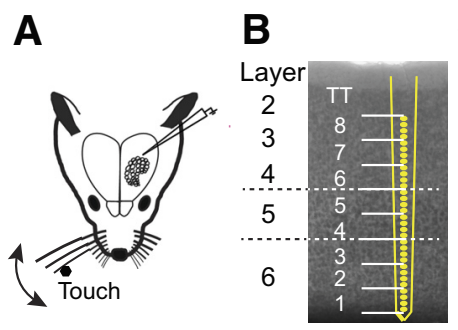

$\mathbf{C}_{\mathrm{WP}}$
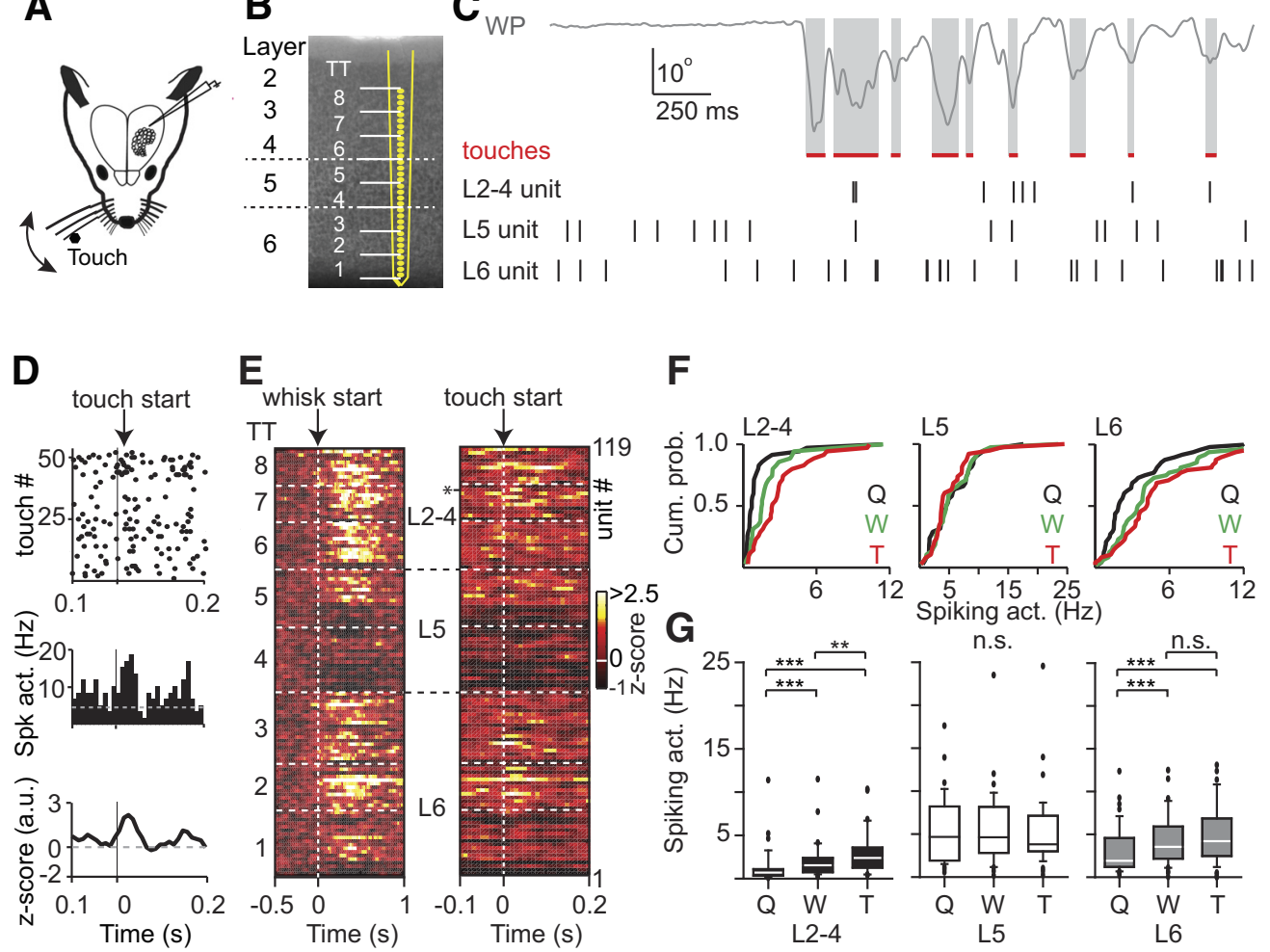

Figure 5. Layer-specific representation in PPC of active whisker touch. $A$, Experimental design for loose-patch and silicon probe recordings across layers of PPC to quantify the correlation between touch and PPC spiking activity. B, Structural layout of the 32-channel silicon probe across PPC layers. White dashed lines indicate borders between L2-4, L5, and L6. C, Snapshot of whisker position (WP) and spikes of three individual example units across PPC layers. D, Raster plot (top), PETH (middle), and z-transformed PETH (bottom) of an example unit from L2 - 4 aligned to touch onset. There is increased spiking activity upon touch. $E$, Heat map of z-transformed spiking activity of all RSUs across PPC layers aligned to whisk start (left) or touch start (right) onset. Each row represents a single unit, arranged according to recording depth. White dashed lines indicate borders between tetrode groups. Asterisk indicates example L2 -4 unit from $\boldsymbol{D}$. F, Layer-specific cumulative distributions to illustrate behavior-dependent modulation of spiking activity. Q, Quiescent; W, whisking; $T$, touch. G, Boxplots represent the population statistics for spiking activity during quiescent, whisking, and touch episodes, respectively. L2 - 4 significantly represents whisking, and spiking activity further increases upon touch (Qvs W: $p<0.001 ; \mathrm{Wvs}$ T: $p<0.01 ; n=35$ ), L5 does not show a change in spiking activity during whisking or touch (Friedman Test, $p=0.51, n=38$ ), and L6 represents whisking but does not show additional modulation upon touch (Q vs W: $p<0.001 ; \mathrm{W}$ vs T: $p>$ $0.05 ; n=48)$. ${ }^{* *} p<0.01,{ }^{* * *} p<0.001$.

activity together with high-speed videography $(200$ or $400 \mathrm{~Hz})$ to track whisker position and object touch (Fig. 5C; Movie 2). Across recording sessions, median touch duration was $40 \mathrm{~ms}$ ( $n=1343$ touch events, first through third quartiles $30-55 \mathrm{~ms}$ ), the interval between touch end and subsequent touch start 140 $\mathrm{ms}$ (first through third quartiles $115-245 \mathrm{~ms}$ ) and interval between consecutive touch starts $230 \mathrm{~ms}$ (first through third quartiles $170-350 \mathrm{~ms}$ ). The short touch duration is similar to behavioral characteristics obtained during tactile exploration in freely moving rats (Hobbs et al., 2015), and similar to the theoretical optimized window of tactile exploration of objects (Bush et al., 2016).

Units were recorded across PPC layers and categorized into RSUs or FSUs based on AP waveform (RSU, $n=121$; FSU, $n=$ 13). We categorized behavior into episodes of quiescence (Q), whisking (W), and object touch (T) and quantified statedependent spiking activity (in $\mathrm{Hz}$ ). Individual units showed heterogeneous responses to whisking or touch, with a subset of units clearly showing a touch-triggered increase in AP spiking (Fig. $5 D)$. Spiking activity across the population was subsequently aligned to either whisking onset or object touch (Fig. 5E).

Upon whisker touch, we found that a subset of units in L2-4 and L6 responded with an increase in spiking activity, whereas units recorded in L5 did not (Fig. 5E, right). Population statistics on absolute spiking activity results in multiple layer- and cell type-specific principles in PPC during whisking and touch (Fig. $5 F, G)$. First, whisking or touch did not change spiking in FSUs

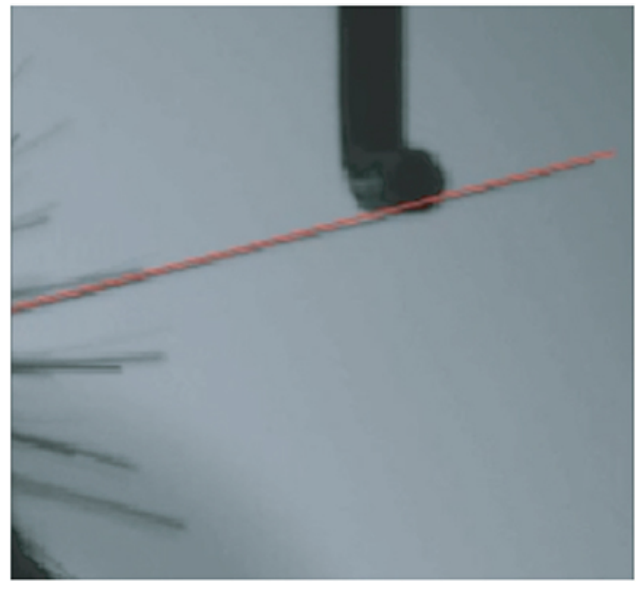

Movie 2. Active object touch video. Video shows an episode of a whisking bout including consecutive active object touches with (in red) the position of the offline tracked single whisker.

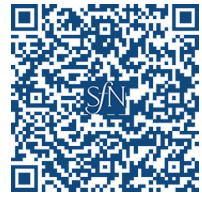

(Table $3 ; p=0.98$ ). In contrast, whisking significantly increased spiking frequency in the population of L2-4 RSUs relative to quiescent episodes. Touch further significantly increased spiking relative to whisking episodes (Q vs $\mathrm{W}: p<0.001$; W vs T: $p<$ 
Table 3. Behavior-dependent modulation of PPC spiking is layer- and cell type-specific $^{a}$

\begin{tabular}{llll}
\hline Layer & Quiescent $(\mathrm{Hz})$ & Whisking $(\mathrm{Hz})$ & Touch $(\mathrm{Hz})$ \\
\hline RSU, 2-4 $(n=35)$ & $0.70(0.37-1.05)$ & $1.54(0.75-2.38)^{* *}$ & $2.39(1.32-3.42)^{*}$ \\
RSU, $5(n=38)$ & $4.67(2.05-8.12)$ & $4.63(2.84-8.07)$ & $3.80(3.03-6.94)$ \\
RSU, 6 $(n=48)$ & $1.91(1.20-4.20)$ & $3.54(2.21-5.80)^{* *}$ & $4.20(2.50-6.73)$ \\
FSU $(n=13)$ & $6.35(3.11-8.59)$ & $4.78(3.25-12.76)$ & $5.30(3.05-9.66)$ \\
\hline
\end{tabular}

${ }^{a}$ Values are median (first through third quartiles).

${ }^{*} p<0.01$; ${ }^{* *} p<0.001$; Quiescent versus Whisking and Whisking versus Touch.

0.01, Friedman Test with Dunn post-test, Fig. 5F,G; Table 3). Second, in L5 RSUs, AP spiking was comparable between quiescent, whisking, and touch episodes (Friedman Test, $p=0.51$ ). Third, in L6 RSUs, we find that whisking significantly increased AP spiking relative to quiescent episodes, but AP spiking during whisking and touch was statistically comparable (Q vs W: $p<$ 0.001 ; W vs T: $p>0.05$, Friedman Test with Dunn post-test; Fig. $5 F, G$; Table 3 ).

To conclude, both whisker sensory and motor information is reliably represented across neurons in PPC but notably with layer-specific differences: whisking is associated with increased spiking in RSU units in L2-4 and L6, and object touch shows increased spiking (relative to whisking) only in RSU units in L2-4.

\section{Discussion}

The PPC is involved in a broad repertoire of cognitive behaviors, including (but not limited to) integration of inputs from multiple sensory modalities as well as motor planning (Andersen, 1997; Andersen et al., 1997; Avillac et al., 2007; Olcese et al., 2013; Licata et al., 2017; Whitlock, 2017; Krumin et al., 2018; Mimica et al., 2018; Nikbakht et al., 2018). Here, we studied suprathreshold processing of unimodal sensorimotor information in rat PPC and present multiple findings: (1) PPC shows functional somatotopy; (2) self-induced whisker motion, specifically whisker movement goals, are represented in L2-4 and L6; (3) these movement goals can be reliably decoded from population activity; and (4) object touch is encoded in L2-4.

We defined PPC as the cortical area posterior to S1, delineated on the mediolateral axis by the borders of the (posteromedial) barrel subfield, and on the anteroposterior axis within $1200 \mu \mathrm{m}$ posterior of the barrel subfield edge. This location corresponds to rostrolateral (RL) and anterior (A) PPC subregions (Wang et al., 2012; Hovde et al., 2019). Our PPC recordings were therefore targeted more lateral compared with a subset of studies (Nitz, 2006, 2012; Wilber et al., 2014, 2017; Hanks et al., 2015), but closely resemble PPC coordinates in others (Kolb and Walkey, 1987; Reep et al., 1994; Whitlock, 2014; Licata et al., 2017; Mimica et al., 2018; Nikbakht et al., 2018). Based on anatomical coordinates, our recordings thus include PPC subdomains 'RL', 'A,' and potentially (part of) 'AM' (Wang et al., 2012; Hovde et al., 2019) and a very small portion of V1. It is, however, almost certain that anatomical borders do not translate one-to-one to discrete functional zones (Olsen and Witter, 2016). Subregion classification exclusively based on function is further complicated by the diversity of behaviors to which PPC activity contributes (Whitlock, 2017). Anatomical borders of V1 and S1 can be revealed relatively straightforward by cytochrome oxidase staining, and PPC is classically defined as the low-intensity strip between strongly stained S1 and V1; but functionally, a gradient may exist along the anteroposterior axis where visual-dominated responses in V1 gradually transform into tactile-dominated responses in S1. In the
PPC territory between visual and tactile cortices, merging of V1 and S1 pathways could facilitate multisensory integration.

Rostrolateral and anterior PPC subregions are commonly referred to as secondary visual areas (Wang and Burkhalter, 2007; Wang et al., 2012; Carandini and Churchland, 2013; Zhuang et al., 2017). Not surprisingly, PPC function in rodents has been studied in the context of visual decision-making (Goard et al., 2016; Licata et al., 2017) but received relatively little attention in the context of active whisker-based somatosensation. It is well known that PPC has reciprocal connections with primary somatosensory (Lee et al., 2011; Wang et al., 2012) and vibrissal motor cortices (Reep et al., 1994; Wilber et al., 2014), which puts PPC in an optimal position to merge neurophysiological correlates of self-generated whisker motion (i.e., efference-copy) and sensory signals from the external world (ex-afferent information) (Cullen, 2004) to guide appropriate behavior. The PPC circuit diagram at cellular resolution remained largely enigmatic so far, and therefore remains speculative which organizational principles and circuit properties lead to layer-specific computational integration of motor and/or sensory information (Petreanu et al., 2009).

We uncovered functional somatotopy in PPC, indicating that tactile processing of whiskers occurs in specialized zones, a wellknown property throughout the whisker sensorimotor pathway and most obvious in primary somatosensory (barrel) cortex (Woolsey and Van der Loos, 1970; Wimmer et al., 2010). Input maps were dense because virtually all recorded locations showed LFP responses after whisker deflection. In contrast, output spiking was sparse $(<1 \mathrm{AP} /$ stim), indicating that the excitation/inhibition balance under anesthetized conditions is unfavorable for AP spiking (Olcese et al., 2013). Functional somatotopy matches organizational principles of S1-PPC feedforward anatomical projections revealed by using anterograde tracer injections (Lee et al., 2011). In addition to the somatotopic organization along the mediolateral axis, we found layer-specific functionality along the radial axis of PPC. First, a passive stimulus evoked the maximal response in L5 relative to L2-4 and L6. During active sensorimotor behavior, voluntary whisking led to increased AP spiking in L2-4 and L6; and whisker object touch further increased this activity, but only for the L2-4 population. The L5 population did not show significant modulation during either whisker motor or sensory processing. This is unexpected in view of the canonical neocortical circuit in which L5 is considered the major output layer of the cortex (Harris and Shepherd, 2015).

In general, neuronal activity in PPC has been shown to correlate to a spectrum of behaviors, including motor behaviors that involve unrestricted exploration and motion/movement planning when subjects are head-fixed or navigate in a virtual reality setting (Espina-Marchant et al., 2006; Cui and Andersen, 2007; Harvey et al., 2012; Hauschild et al., 2012; Mimica et al., 2018; Crochet et al., 2019). We studied the functional architecture of PPC during tactile sensorimotor behavior in head-fixed rats, and the temporal dynamics of whisking and object touch resemble those observed in freely moving conditions (Carvell and Simons, 1990; Hobbs et al., 2015). The full spectrum of cell type-specific activity in PPC will probably only emerge, however, during complex tasks in a behavioral arena that allows unrestricted navigation. The correlation between spiking activity and behavioral complexity may be particularly relevant for cortical output layer 5 , which is occupied by intratelencephalic and pyramidal tract neurons (Harris and Shepherd, 2015; Zeng and Sanes, 2017). The pyramidal tract neurons project to multiple subcortical targets and orchestrate behavioral output (Helmstaedter et al., 2007; 
Kim et al., 2015; Rojas-Piloni et al., 2017). In anterior lateral motor cortex, for instance, spiking activity in these L5 pyramidal tract neurons causally encodes upcoming movements (Li et al., 2015; Economo et al., 2018). We cannot exclude the possibility that lack of L5 modulation in PPC during exploratory whisking and naive object touch is related to head-fixed conditions. An alternative hypothesis, however, is that neocortical pyramidal tract neurons have region-specific (Fletcher and Williams, 2019) or task-specific functions (Mel et al., 2017) and PPC L5 is only recruited during more challenging behaviors, for instance, during task-learning or coupling of multisensory perception to appropriate action (Olcese et al., 2013; Krumin et al., 2018; Nikbakht et al., 2018). It also remains to be determined whether PPC L5 is, analogous to anterior lateral motor cortex L5, directly involved in motor output (Li et al., 2015). Our results from headfixed rats thus provide a valuable step toward understanding cell type- and layer-specific function in PPC during tactile processing, but it will be appealing to study layer-specific coding principles in PPC during more challenging behavioral conditions to reach a more comprehensive view on layer specificity of PPC input-output computations (Lee et al., 2011).

Because PPC receives little input from somatosensory thalamic nuclei (Wilber et al., 2014), intracortical pathways are most realistic sources to relay information on whisker motion and touch to PPC. The anatomical input projections carrying efference-copy information to PPC could originate directly from motor cortices, but bulk-labeling approaches make it impossible to formulate hypotheses on layer specificity during coding of motor behavior. A different source for efference-copy coding could be S1, which projects monosynaptically to PPC (Lee et al., 2011). In S1, PPC-projecting neurons are found across layers 2-5 and are preferentially located in septal regions (Lee et al., 2011). These S1-septal regions have been associated with the paralemniscal pathway (Alloway, 2008), dedicated to coding of whisker motion during active sensing (Ahissar et al., 2000; Yu et al., 2006; but see Urbain et al., 2015; Moore et al., 2015b). The S1-L5A (slender tufted) pyramids deserve focused attention because this cell type has been shown to encode whisker self-motion (de Kock and Sakmann, 2009), and a subset of these neurons directly project to PPC, albeit with little specificity with respect to layers (Oberlaender et al., 2011, Fig. S1, fourth example). One potential strategy for future experiments to uncover the causal relationship between specific inputs and coding principles in PPC is to manipulate spiking activity of morphologically identified S1 cell types during whisker-guided sensorimotor behavior and determine the outcome on PPC spiking activity. Similarly, the excitatory inputs leading to increased output spiking upon whisker touch remain speculative and could be investigated through manipulation of possible input sources, which may be a combination of nonsensory thalamic inputs (para)lemniscal inputs from barrel column- and septum-associated neurons across S1 layers or even alternative cortical pathways.

In conclusion, we uncovered somatotopy in PPC, revealed layer-specific sensorimotor processing, and showed that PPC spiking predicts whisker movement in awake, behaving rats. This opens the path to design additional experiments to reveal cell type-specific contributions to sensorimotor processing. When we apply these to somatosensory, motor, and additional association areas, the sensorimotor loop may be closed to advance the coding and decoding algorithms of neuronal circuits underlying sensory-guided motor output. These algorithms will in turn push the development of PPC-based neuro-prosthetic applications to restore sensorimotor behavior after loss of function (Hauschild et al., 2012; Bensmaia and Miller, 2014; Andersen et al., 2014; Aflalo et al., 2015).

\section{References}

Aflalo T, Kellis S, Klaes C, Lee B, Shi Y, Pejsa K, Shanfield K, Hayes-Jackson S, Aisen M, Heck C, Liu C, Andersen RA (2015) Neurophysiology: decoding motor imagery from the posterior parietal cortex of a tetraplegic human. Science 348:906-910.

Ahissar E, Sosnik R, Haidarliu S (2000) Transformation from temporal to rate coding in a somatosensory thalamocortical pathway. Nature 406:302-306.

Alloway KD (2008) Information processing streams in rodent barrel cortex: the differential functions of barrel and septal circuits. Cereb Cortex 18:979-989.

Andersen RA (1997) Multimodal integration for the representation of space in the posterior parietal cortex. Philos Trans R Soc Lond B Biol Sci 352:1421-1428

Andersen RA, Snyder LH, Bradley DC, Xing J (1997) Multimodal representation of space in the posterior parietal cortex and its use in planning movements. Annu Rev Neurosci 20:303-330.

Andersen RA, Kellis S, Klaes C, Aflalo T (2014) Toward more versatile and intuitive cortical brain-machine interfaces. Curr Biol 24:R885-R897.

Avillac M, Ben Hamed S, Duhamel JR (2007) Multisensory integration in the ventral intraparietal area of the macaque monkey. J Neurosci 27:19221932.

Barthó P, Hirase H, Monconduit L, Zugaro M, Harris KD, Buzsáki G (2004) Characterization of neocortical principal cells and interneurons by network interactions and extracellular features. J Neurophysiol 92:600-608.

Bensmaia SJ, Miller LE (2014) Restoring sensorimotor function through intracortical interfaces: progress and looming challenges. Nat Rev Neurosci 15:313-325.

Boudewijns ZS, Groen MR, Lodder B, McMaster MT, Kalogreades L, de Haan R, Narayanan RT, Meredith RM, Mansvelder HD, de Kock CP (2013) Layer-specific high-frequency action potential spiking in the prefrontal cortex of awake rats. Front Cell Neurosci 7:99.

Bucci DJ (2009) Posterior parietal cortex: an interface between attention and learning? Neurobiol Learn Mem 91:114-120.

Bush NE, Solla SA, Hartmann MJ (2016) Whisking mechanics and active sensing. Curr Opin Neurobiol 40:178-188.

Carandini M, Churchland AK (2013) Probing perceptual decisions in rodents. Nat Neurosci 16:824-831.

Carvell GE, Simons DJ (1990) Biometric analyses of vibrissal tactile discrimination in the rat. J Neurosci 10:2638-2648.

Chagnac-Amitai Y, Luhmann HJ, Prince DA (1990) Burst generating and regular spiking layer 5 pyramidal neurons of rat neocortex have different morphological features. J Comp Neurol 296:598-613.

Chen LL, Lin LH, Barnes CA, McNaughton BL (1994) Head-direction cells in the rat posterior cortex: II. Contributions of visual and ideothetic information to the directional firing. Exp Brain Res 101:24-34.

Clack NG, O'Connor DH, Huber D, Petreanu L, Hires A, Peron S, Svoboda K, Myers EW (2012) Automated tracking of whiskers in videos of head fixed rodents. PLoS Comput Biol 8:e1002591.

Crochet S, Lee SH, Petersen CC (2019) Neural circuits for goal-directed sensorimotor transformations. Trends Neurosci 42:66-77.

Csicsvari J, Hirase H, Czurkó A, Mamiya A, Buzsáki G (1999) Oscillatory coupling of hippocampal pyramidal cells and interneurons in the behaving rat. J Neurosci 19:274-287.

Cui H, Andersen RA (2007) Posterior parietal cortex encodes autonomously selected motor plans. Neuron 56:552-559.

Cullen KE (2004) Sensory signals during active versus passive movement. Curr Opin Neurobiol 14:698-706.

Defelipe J (2011) The evolution of the brain, the human nature of cortical circuits, and intellectual creativity. Front Neuroanat 5:29.

de Kock CP (2016) Juxtasomal loose-patch recordings in awake, head-fixed rats to study the link between structure and function of individual neurons. In: Advanced patch-clamp analysis for neuroscientists. New York: Springer Protocols.

de Kock CP, Sakmann B (2009) Spiking in primary somatosensory cortex during natural whisking in awake head-restrained rats is cell type-specific. Proc Natl Acad Sci U S A 106:16446-16450.

de Kock CP, Bruno RM, Spors H, Sakmann B (2007) Layer and cell type 
specific suprathreshold stimulus representation in primary somatosensory cortex. J Physiol 581:139-154.

Economo MN, Viswanathan S, Tasic B, Bas E, Winnubst J, Menon V, Graybuck LT, Nguyen TN, Smith KA, Yao Z, Wang L, Gerfen CR, Chandrashekar J, Zeng H, Looger LL, Svoboda K (2018) Distinct descending motor cortex pathways and their roles in movement. Nature 563:79-84.

Egger R, Narayanan RT, Helmstaedter M, de Kock CP, Oberlaender M (2012) 3D reconstruction and standardization of the rat vibrissal cortex for precise registration of single neuron morphology. PLoS Comput Biol 8:e1002837.

Espina-Marchant P, Pinto-Hamuy T, Bustamante D, Morales P, Robles L, Herrera-Marschitz M (2006) Spatial cognition and memory: a reversible lesion with lidocaine into the anteromedial/posterior parietal cortex (AM/PPC) affects differently working and long-term memory on two foraging tasks. Biol Res 39:601-609.

Feldmeyer D, Brecht M, Helmchen F, Petersen CC, Poulet JF, Staiger JF, Luhmann HJ, Schwarz C (2013) Barrel cortex function. Prog Neurobiol 103:3-27.

Fletcher LN, Williams SR (2019) Neocortical topology governs the dendritic integrative capacity of layer 5 pyramidal neurons. Neuron 101:76-90.e4.

Fraley C, Raftery AE (2002) Model-based clustering, discriminant analysis, and density estimation. J Am Stat Assoc 97:611-631.

Fraley C, Raftery AE (2003) Enhanced model-based clustering, density estimation, and discriminant analysis software: MCLUST. J Classif 20:263286.

Goard MJ, Pho GN, Woodson J, Sur M (2016) Distinct roles of visual, parietal, and frontal motor cortices in memory-guided sensorimotor decisions. eLife 5:e13764.

Guo ZV, Li N, Huber D, Ophir E, Gutnisky D, Ting JT, Feng G, Svoboda K (2014) Flow of cortical activity underlying a tactile decision in mice. Neuron 81:179-194.

Hanks TD, Kopec CD, Brunton BW, Duan CA, Erlich JC, Brody CD (2015) Distinct relationships of parietal and prefrontal cortices to evidence accumulation. Nature 520:220-223.

Harris KD, Shepherd GM (2015) The neocortical circuit: themes and variations. Nat Neurosci 18:170-181.

Harris KD, Henze DA, Csicsvari J, Hirase H, Buzsáki G (2000) Accuracy of tetrode spike separation as determined by simultaneous intracellular and extracellular measurements. J Neurophysiol 84:401-414.

Harris KD, Hirase H, Leinekugel X, Henze DA, Buzsáki G (2001) Temporal interaction between single spikes and complex spike bursts in hippocampal pyramidal cells. Neuron 32:141-149.

Harris KD, Quiroga RQ, Freeman J, Smith SL (2016) Improving data quality in neuronal population recordings. Nat Neurosci 19:1165-1174.

Harvey CD, Coen P, Tank DW (2012) Choice-specific sequences in parietal cortex during a virtual-navigation decision task. Nature 484:62-68.

Hauschild M, Mulliken GH, Fineman I, Loeb GE, Andersen RA (2012) Cognitive signals for brain-machine interfaces in posterior parietal cortex include continuous 3D trajectory commands. Proc Natl Acad Sci U S A 109:17075-17080.

Helmstaedter M, de Kock CP, Feldmeyer D, Bruno RM, Sakmann B (2007) Reconstruction of an average cortical column in silico. Brain Res Rev 55:193-203.

Herfst L, Burgalossi A, Haskic K, Tukker JJ, Schmidt M, Brecht M (2012) Friction-based stabilization of juxtacellular recordings in freely moving rats. J Neurophysiol 108:697-707.

Hires SA, Efros AL, Svoboda K (2013) Whisker dynamics underlying tactile exploration. J Neurosci 33:9576-9591.

Hobbs JA, Towal RB, Hartmann MJ (2015) Spatiotemporal patterns of contact across the rat vibrissal array during exploratory behavior. Front Behav Neurosci 9:356.

Horikawa K, Armstrong WE (1988) A versatile means of intracellular labeling: injection of biocytin and its detection with avidin conjugates. J Neurosci Methods 25:1-11.

Hovde K, Gianatti M, Witter MP, Whitlock JR (2019) Architecture and organization of mouse posterior parietal cortex relative to extrastriate areas. Eur J Neurosci 49:1313-1329.

Kim EJ, Juavinett AL, Kyubwa EM, Jacobs MW, Callaway EM (2015) Three types of cortical layer 5 neurons that differ in brain-wide connectivity and function. Neuron 88:1253-1267.

Knutsen PM, Derdikman D, Ahissar E (2005) Tracking whisker and head movements in unrestrained behaving rodents. J Neurophysiol 93:2294-2301.

Kolb B, Walkey J (1987) Behavioural and anatomical studies of the posterior parietal cortex in the rat. Behav Brain Res 23:127-145.

Kortelainen J, Väyrynen E, Jia X, Seppänen T, Thakor N (2012) EEG-based detection of awakening from isoflurane anesthesia in rats. Conf Proc IEEE Eng Med Biol Soc 2012:4279-4282.

Krumin M, Lee JJ, Harris KD, Carandini M (2018) Decision and navigation in mouse parietal cortex. eLife 7:e42583.

Lee T, Alloway KD, Kim U (2011) Interconnected cortical networks between primary somatosensory cortex septal columns and posterior parietal cortex in rat. J Comp Neurol 519:405-419.

Le Merre P, Esmaeili V, Charrière E, Galan K, Salin PA, Petersen CC, Crochet S (2018) Reward-based learning drives rapid sensory signals in medial prefrontal cortex and dorsal hippocampus necessary for goal-directed behavior. Neuron 97:83-91.e5.

Li N, Chen TW, Guo ZV, Gerfen CR, Svoboda K (2015) A motor cortex circuit for motor planning and movement. Nature 519:51-56.

Licata AM, Kaufman MT, Raposo D, Ryan MB, Sheppard JP, Churchland AK (2017) Posterior parietal cortex guides visual decisions in rats. J Neurosci 37:4954-4966.

McNaughton BL, Mizumori SJ, Barnes CA, Leonard BJ, Marquis M, Green EJ (1994) Cortical representation of motion during unrestrained spatial navigation in the rat. Cereb Cortex 4:27-39.

Mel BW, Schiller J, Poirazi P (2017) Synaptic plasticity in dendrites: complications and coping strategies. Curr Opin Neurobiol 43:177-186.

Mimica B, Dunn BA, Tombaz T, Bojja V, Whitlock JR (2018) Efficient cortical coding of 3D posture in freely behaving rats. Science 362:584 -589 .

Mohan H, de Haan R, Mansvelder HD, de Kock CP (2018) The posterior parietal cortex as integrative hub for whisker sensorimotor information. Neuroscience 368:240-245.

Moore JD, Deschenes M, Kleinfeld D (2015a) Juxtacellular monitoring and localization of single neurons within sub-cortical brain structures of alert, head-restrained rats. J Vis Exp 2015:98.

Moore JD, Mercer Lindsay N, Deschênes M, Kleinfeld D (2015b) Vibrissa self-motion and touch are reliably encoded along the same somatosensory pathway from brainstem through thalamus. PLoS Biol 13:e1002253.

Narayanan RT, Mohan H, Broersen R, de Haan R, Pieneman AW, de Kock CP (2014) Juxtasomal biocytin labeling to study the structure-function relationship of individual cortical neurons. J Vis Exp 84:e51359.

Nikbakht N, Tafreshiha A, Zoccolan D, Diamond ME (2018) Supralinear and supramodal integration of visual and tactile signals in rats: psychophysics and neuronal mechanisms. Neuron 97:626-639.e8.

Nitz DA (2006) Tracking route progression in the posterior parietal cortex. Neuron 49:747-756.

Nitz DA (2012) Spaces within spaces: rat parietal cortex neurons register position across three reference frames. Nat Neurosci 15:1365-1367.

Oberlaender M, Boudewijns ZS, Kleele T, Mansvelder HD, Sakmann B, de Kock CP (2011) Three-dimensional axon morphologies of individual layer 5 neurons indicate cell type-specific intracortical pathways for whisker motion and touch. Proc Natl Acad Sci U S A 108:4188-4193.

Olcese U, Iurilli G, Medini P (2013) Cellular and synaptic architecture of multisensory integration in the mouse neocortex. Neuron 79:579-593.

Olsen GM, Witter MP (2016) Posterior parietal cortex of the rat: architectural delineation and thalamic differentiation. J Comp Neurol 524: 3774-3809.

Petersen CC (2007) The functional organization of the barrel cortex. Neuron 56:339-355.

Petreanu L, Mao T, Sternson SM, Svoboda K (2009) The subcellular organization of neocortical excitatory connections. Nature 457:1142-1145.

Pinault D (1996) A novel single-cell staining procedure performed in vivo under electrophysiological control: morpho-functional features of juxtacellularly labeled thalamic cells and other central neurons with biocytin or neurobiotin. J Neurosci Methods 65:113-136.

Reep RL, Chandler HC, King V, Corwin JV (1994) Rat posterior parietal cortex: topography of corticocortical and thalamic connections. Exp Brain Res 100:67-84.

Rojas-Piloni G, Guest JM, Egger R, Johnson AS, Sakmann B, Oberlaender M (2017) Relationships between structure, in vivo function and long-range axonal target of cortical pyramidal tract neurons. Nat Commun 8:870.

Sigl-Glöckner J, Brecht M (2017) Polyploidy and the cellular and areal diversity of rat cortical layer 5 pyramidal neurons. Cell Rep 20:2575-2583. 
Tomassy GS, Berger DR, Chen HH, Kasthuri N, Hayworth KJ, Vercelli A, Seung HS, Lichtman JW, Arlotta P (2014) Distinct profiles of myelin distribution along single axons of pyramidal neurons in the neocortex. Science 344:319-324.

Towal RB, Hartmann MJ (2008) Variability in velocity profiles during free-air whisking behavior of unrestrained rats. J Neurophysiol 100: $740-752$.

Urbain N, Salin PA, Libourel PA, Comte JC, Gentet LJ, Petersen CC (2015) Whisking-related changes in neuronal firing and membrane potential dynamics in the somatosensory thalamus of awake mice. Cell Rep 13:647-656.

Wang Q, Burkhalter A (2007) Area map of mouse visual cortex. J Comp Neurol 502:339-357.

Wang Q, Sporns O, Burkhalter A (2012) Network analysis of corticocortical connections reveals ventral and dorsal processing streams in mouse visual cortex. J Neurosci 32:4386-4399.

Whitlock JR (2014) Navigating actions through the rodent parietal cortex. Front Hum Neurosci 8:293.

Whitlock JR (2017) Posterior parietal cortex. Curr Biol 27:R691-R695.

Wilber AA, Clark BJ, Demecha AJ, Mesina L, Vos JM, McNaughton BL
(2014) Cortical connectivity maps reveal anatomically distinct areas in the parietal cortex of the rat. Front Neural Circuits 8:146.

Wilber AA, Skelin I, Wu W, McNaughton BL (2017) Laminar organization of encoding and memory reactivation in the parietal cortex. Neuron 95:1406-1419.e5.

Wimmer VC, Bruno RM, de Kock CP, Kuner T, Sakmann B (2010) Dimensions of a projection column and architecture of VPM and POm axons in rat vibrissal cortex. Cereb Cortex 20:2265-2276.

Wong-Riley M (1979) Changes in the visual system of monocularly sutured or enucleated cats demonstrable with cytochrome oxidase histochemistry. Brain Res 171:11-28.

Woolsey TA, Van der Loos H (1970) The structural organization of layer IV in the somatosensory region (SI) of mouse cerebral cortex: the description of a cortical field composed of discrete cytoarchitectonic units. Brain Res 17:205-242.

Yu C, Derdikman D, Haidarliu S, Ahissar E (2006) Parallel thalamic pathways for whisking and touch signals in the rat. PLoS Biol 4:e124.

Zeng H, Sanes JR (2017) Neuronal cell type classification: challenges, opportunities and the path forward. Nat Rev Neurosci 18:530-546.

Zhuang J, Ng L, Williams D, Valley M, Li Y, Garrett M, Waters J (2017) An extended retinotopic map of mouse cortex. eLife 6:e18372. 